\title{
Pseudo-synchronous system for recording action and field potentials simultaneously
}

\author{
Chia-Nan Chien ${ }^{\text {a }}$, Jen-Yu Li ${ }^{\text {b }}$, Fu-Shan Jaw ${ }^{\text {a,* }}$ \\ ${ }^{a}$ Institute of Biomedical Engineering, National Taiwan University, Taipei, Taiwan \\ b Department of Electrical Engineering, National Taiwan University, Taipei, Taiwan
}

Received 24 October 2005; received in revised form 26 June 2006; accepted 28 June 2006

Available online 25 July 2006

\begin{abstract}
Large-scale simultaneous recording could help us to understand the complex behavior of neuronal ensembles. Field potentials (FPs) and multi-unit activities (MUAs) are to complement each other. Here, a hybrid multichannel system was proposed to record FPs and MUAs. Firstly, a three-stage signal-conditioning unit was designed that offered low noise, low cost and excellent line-driving capabilities. The parallel architecture of the filter stage, just before the analog-to-digital (A/D) converter card, allowed the MUA and FPs to be recorded simultaneously without further signal processing. Secondly, to obtain a precision map, a 64-channel high-speed A/D card and its software program were purchased, which allowed "pseudo-synchronous" acquisition among different channels. Finally, a practical application of this system in investigating cortical responses showed that it met the requirements of ensemble recording. The detailed design considerations and methods for implementing the system could be valuable to other neuroscience laboratories.
\end{abstract}

(c) 2006 Elsevier Ltd. All rights reserved.

Keywords: Amplifier; Data-acquisition; Field potential; Multichannel; Multi-unit action potentials

\section{Introduction}

The processing of somatosensory information in the mammalian brain involves the transmission of neural activities from the skin to the neocortex through parallel pathways, which ascend through a hierarchical sequence of neural structures [1,2]. Even the simplest response will depend on the synchronous activation of neuron populations $[3,4]$. Multichannel recording is thus becoming indispens-

\footnotetext{
* Corresponding author. Tel.: +886 2 23687401; fax: +8862 33665268.

E-mail address: jaw@ha.mc.ntu.edu.tw (F.-S. Jaw).
}

able. The recording of field potentials (FPs) and multi-unit activities (MUAs) have been used to observe the activities of groups of neurons and provide information about the distribution and functions [5,6]. FPs provide the macrocosm and MAUs give the microcosmic of the brain activities, they are to complement each other.

Fortunately, recent advances in micromachine multichannel probes have made large-scale recording of neural ensembles feasible [7,8]. Modern high performance signal-conditioning and data-acquisition systems have further advanced the application of multichannel recording techniques [9-11]. However, so many manufacturers and publications of 
multichannel system that could be recorded only alone either FPs or MAUs.

The current paper details a complete multichannel system, which can easy and rapid to be constructed, including a 16-channel preamplifier, two 16-channel filters, a 64-channel analog-to-digital (A/D) converter card, and a user interface. Novel ideas are suggested concerning the line-driving capability of the amplifiers, the parallel architecture of the filters and "pseudo-synchronous" acquisition for the sampling of neuronal signals. Furthermore, this system can record MUAs as well as FPs concurrently. A cortical evoked response is used to illustrate its utility. The design considerations for each module provide detailed information about the construction of a multichannel system for neural activities recording. We believe that this information will be useful for other neuroscience laboratories.

\section{System construction}

\subsection{Design considerations}

As a complete multichannel recording system would have been expensive, complicated and application specific, several factors were carefully considered from the outset of the study. The first question was whether to build or to buy? This led us to think about the nature of the functional blocks that would make up the complete system. In principle, a multichannel microelectrode, a signal-conditioning unit (usually comprising amplifiers and filters) and a data-acquisition system are the essential parts of this type of system.

The specification of a multichannel electrode depends not only on the technologies available, but also on the characteristics of the recording target; in other words, the major factor that affects the configuration of a multichannel electrode is the recording site of the neuronal signals. However, multichannel microelectrodes purchased from commercial manufacturers or academic laboratories are suitable for many experimental paradigms [12-15].

The second problem is determining the architecture or composition of the signal-conditioning unit. The major consideration for a multichannel conditioning unit is its performance. Fig. 1 illustrates the proposed architecture. Instead of the time-multiplex technique [16], parallel architecture was used to avoid the problem of a long settling time between channel switching.

Considering the potentially large number of channels, the data-acquisition system deserves close attention. For biomedical applications, the signal sampling should be simultaneous among the channels [17] and closely synchronized with external events [18]. Currently, the maximum number of simultaneous channels for a commercial A/D card is eight. Therefore, to increase the number of input channels, the "pseudo-synchronous" technique was used [19]. The necessary hardware development is too complex to be completed in a short time. Furthermore, it would have been impractical to develop a user interface within the given time restrictions. Therefore, the PCI-6071E A/D data-acquisition card and associated software was purchased. This card features 64 analog inputs and can stream data to disk at a rate of up to $1.25 \mathrm{M}$ words per second. For instance, with a 16-channel application, the maximum sampling rate of each channel is $\sim 78 \mathrm{kHz}$ under idealized conditions. Finally, a Pentium III $(1 \mathrm{GHz})$ computer was used as the platform

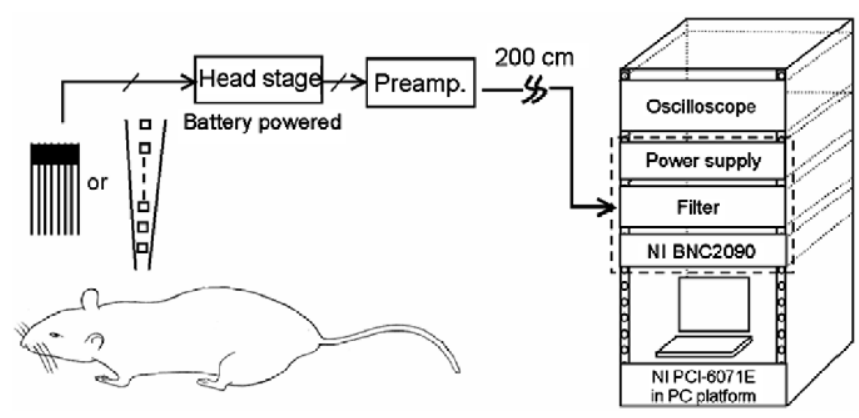

Fig. 1. Complete system architecture. Each channel of the signal-conditioning unit consisted of a head stage, a preamplifier and two filters. For low noise interference, the head stage was placed close to the microelectrode and was battery powered. The preamplifier was placed beside the stereotaxic apparatus and was far away from the instrument frame. The filters were rack mounted as close as possible to the inputs of the A/D converter card. 
for the graphic user interface, which was developed using the LabVIEW programming language.

\subsection{Implementation of the signal-conditioning unit}

As illustrated in Fig. 1, the signal-conditioning unit was separated into the headstage, preamplifier, and filter sections. The detailed circuits are shown in Fig. 2. Usually the headstage was integrated into the multichannel electrode for noise reduction. For each channel, an ultra-low-power operational amplifier $(\mathrm{OP} ; 1 / 4 \mathrm{OP} 481 ; 4 \mu \mathrm{A})$ was used as a voltage follower for impedance conversion.

Because the preamplifier was the first gain stage, its characteristics were crucial to the overall performance of the system. As the headstage consumed relatively little power, the preamplifier could not be placed a large distance away. In fact, the distance
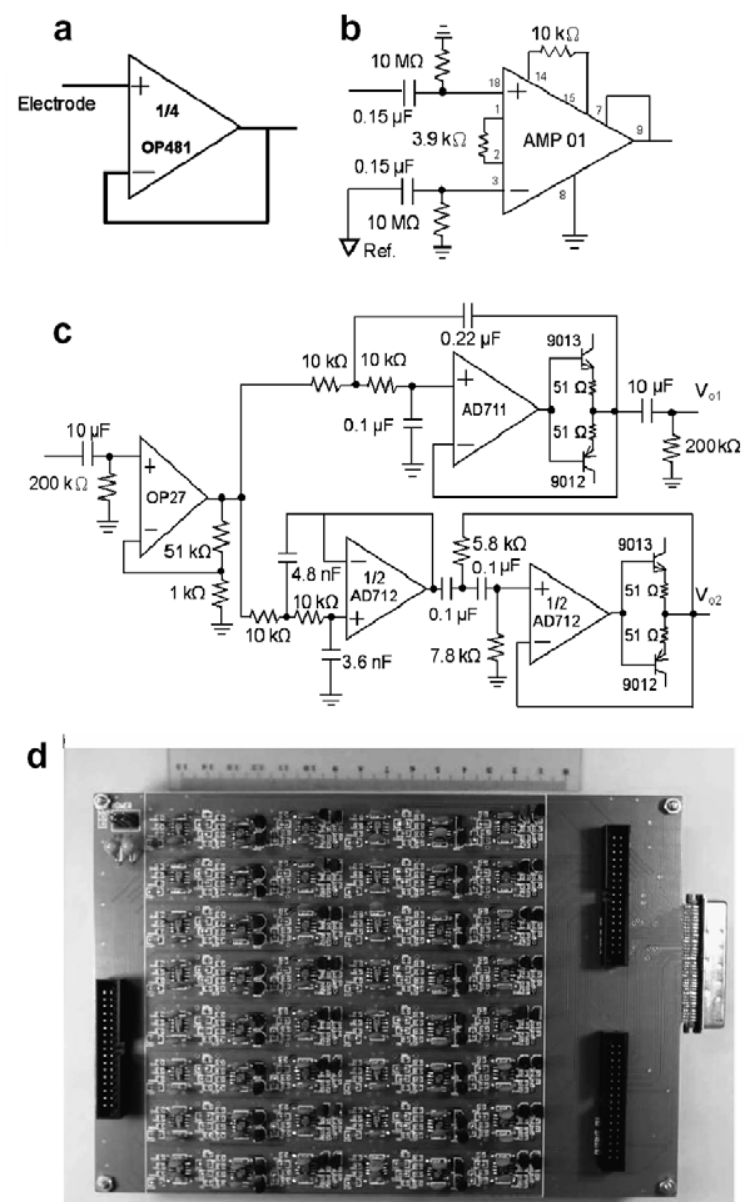

Fig. 2. Circuit diagram of the signal-conditioning unit. Only one channel of the head stage (a), the preamplifier (b) and the filters (c) is shown for simplicity. A photograph of the finished filter module is shown in (d). between them was kept within $1 \mathrm{~m}$. Therefore, the requirements of the preamplifier included low noise, a high common-mode rejection ratio (CMRR) and an excellent line-driving capability to accommodate the capacitive loading of the long cable connecting to the filter stage, which was mounted on the instrument frame [20] could be achieved. In addition to its electrical specifications, the size and cost-per-channel needed to be kept as low as possible because many channels were to be utilized. Thus, the instrumentation amplifier (IA)-AMP01 (Analog devices) - that could fulfill all of these requirements was selected to implement one channel of this stage [21]. As a result of the current-feedback architecture, this IA had a high CMRR $(>125 \mathrm{~dB}$; gain $=1000)$, low noise performance $(15 \mathrm{nV} / \sqrt{ } \mathrm{Hz})$ and excellent driving capabilities $(60 \mathrm{~mA} / \pm 15 \mathrm{~V})$, which made it the best candidate for our purpose. The gain of this stage was programmed as 50 .

The final component of the signal-conditioning unit was the filter. A fixed gain of 50 was adopted in this stage to make an overall gain of 2500 . Also, a low-noise OP (OP27) was used to amplify the signal. A general-purpose OP (AD711) was used to implement a second-order Butterworth low-pass filter. Two of these amplifiers were used to construct a second-order Bessel band-pass filter. The dual-inline package version (AD712) was used to conserve space. The Butterworth and Bessel filters were utilized for the FPs and the MUA applications, respectively [22]. They were arranged in parallel after the gain stage. Because the connection cable of the A/D card was 2-m long, a push-pull output stage (2N9012 and 2N9013) was incorporated into the feedback loop of the OPs for current boosting.

\subsection{Data-acquisition unit}

The data-acquisition portion of the multichannel system plays a key role in ensemble recordings. To utilize the power of digital signal processing for $2 \mathrm{D}$ or $3 \mathrm{D}$ mappings, a high performance $\mathrm{A} / \mathrm{D}$ card is necessary. Thus, a high-speed 64-channel $\mathrm{A} / \mathrm{D}$ card (PCI-6071E) and its operating environment (the LabVIEW programming language) were purchased in order to implement the data-acquisition system. To map the responses in a $2 \mathrm{D}$ or $3 \mathrm{D}$ region accurately, the multiplexing delay between channels should be kept to a minimum. As a conversional concept, a low-speed A/D card is adequate to sample the slow-varying FPs. However, as the number of channels increases, the latency between the first 
a
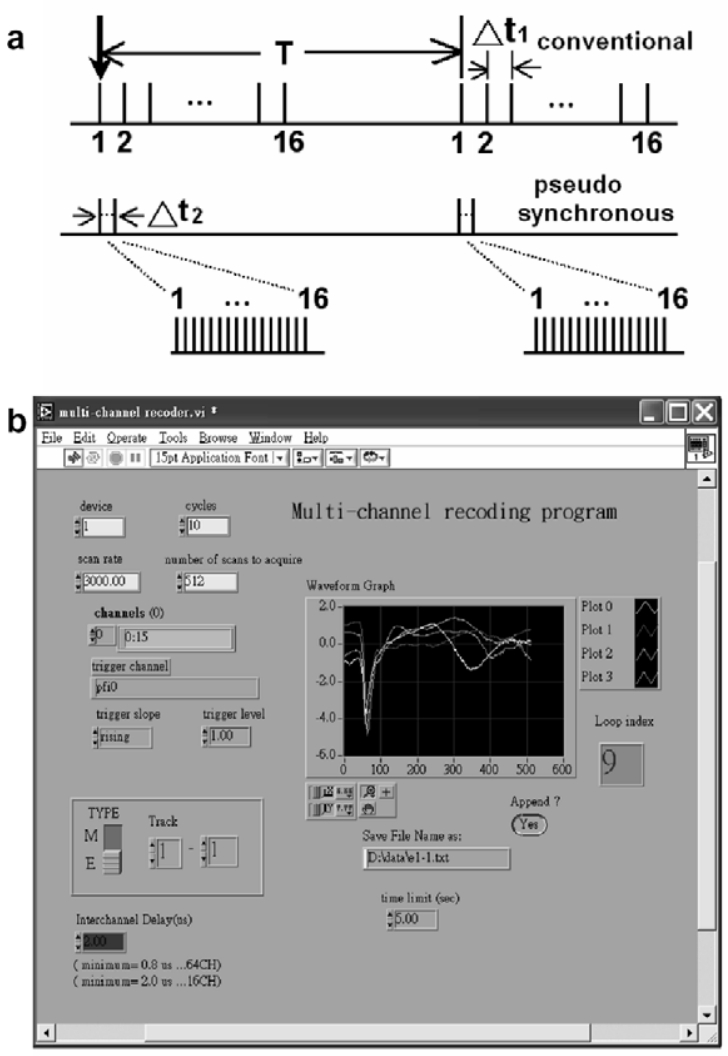

Fig. 3. Principle of pseudo-synchronous acquisition (a) and the graphic user interface (b) of the data-acquisition system.

and the last channels can rise to an unacceptable level (as shown in Fig. 3a). The concept of pseudo-synchronous acquisition was proposed to solve this problem. Fig. 3a shows the difference between the conventional method (upper trace) and the proposed approach (lower trace). The sampling rate of the FPs does not necessary very high that only have to satisfy the sampling theorem; for example, in this case it is $\sim 1 \mathrm{kHz}$. If 16 channels of the FPs are to be acquired at $1 \mathrm{kHz}(1 / T)$ each, an $\mathrm{A} / \mathrm{D}$ card sampling at $20 \mathrm{kHz}\left(1 / \Delta t_{1}\right)$ could be used in conversional acquisition. In this case, the latency between the first and the last channel is $0.75 \mathrm{~ms}$. By contrast, we can digitize the 16 channels of the FPs at $1 \mathrm{MHz}$ (the pseudo-synchronous method) and limit this latency to $15 \mu \mathrm{s}\left(\Delta t_{2}\right)$. As the minimal latency between two consecutive channels of the PCI-6071E is only $0.8 \mu \mathrm{s}$, the characteristics of the pseudo-synchronous method can be fulfilled.

To facilitate the use of this system, a graphic user interface was developed, as shown in Fig. 3b. Users could configure the sampling rate, the latency between two consecutive channels, the number of cycles for signal averaging and the data points per cycle, and could simultaneously monitor the acquired signals online.

\section{Practical application of the system}

The frequency response of the preamplifier is plotted in Fig. 4a. The cutoff frequency was $105 \mathrm{kHz}$ with a measured gain of $\sim 51.2$. The frequency responses of the filters are shown in Fig. $4 \mathrm{~b}$ and $\mathrm{c}$ : the former is the response of the
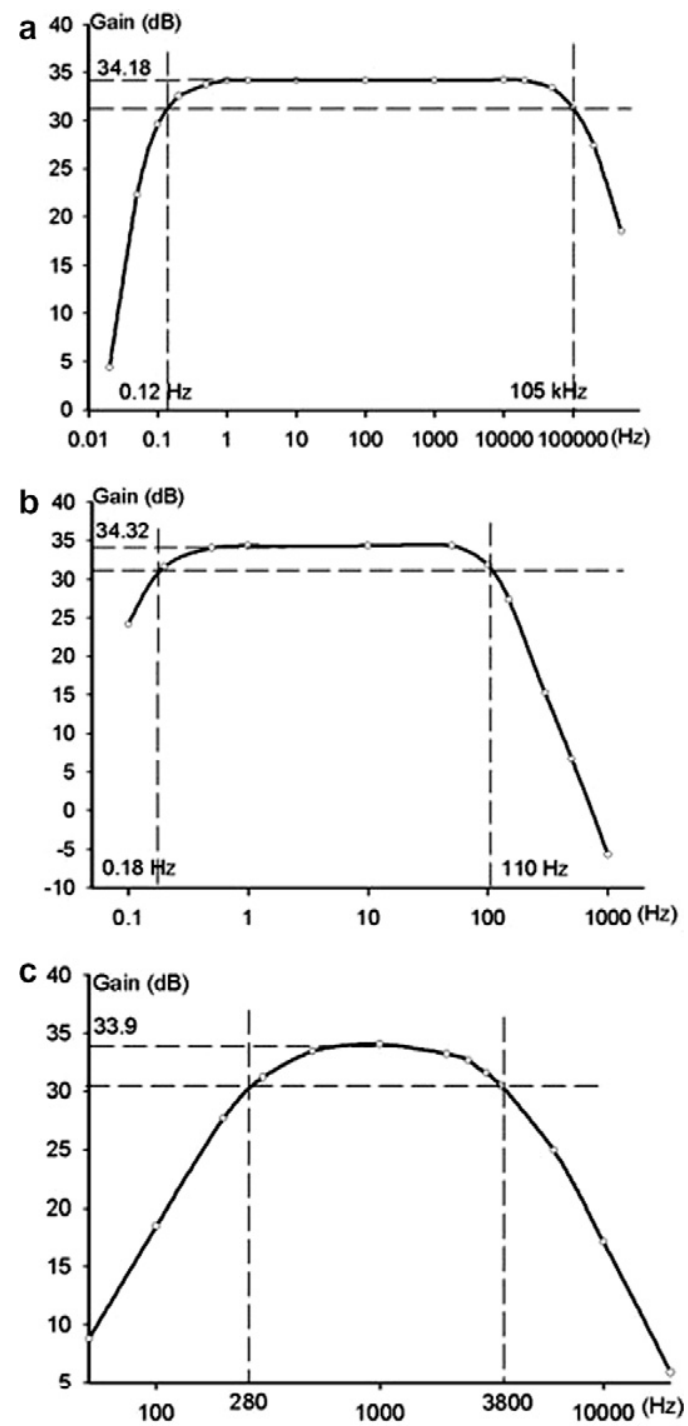

Fig. 4. The frequency responses of the preamplifier stage (a), the Butterworth (b) and the Bessel filters (c). 

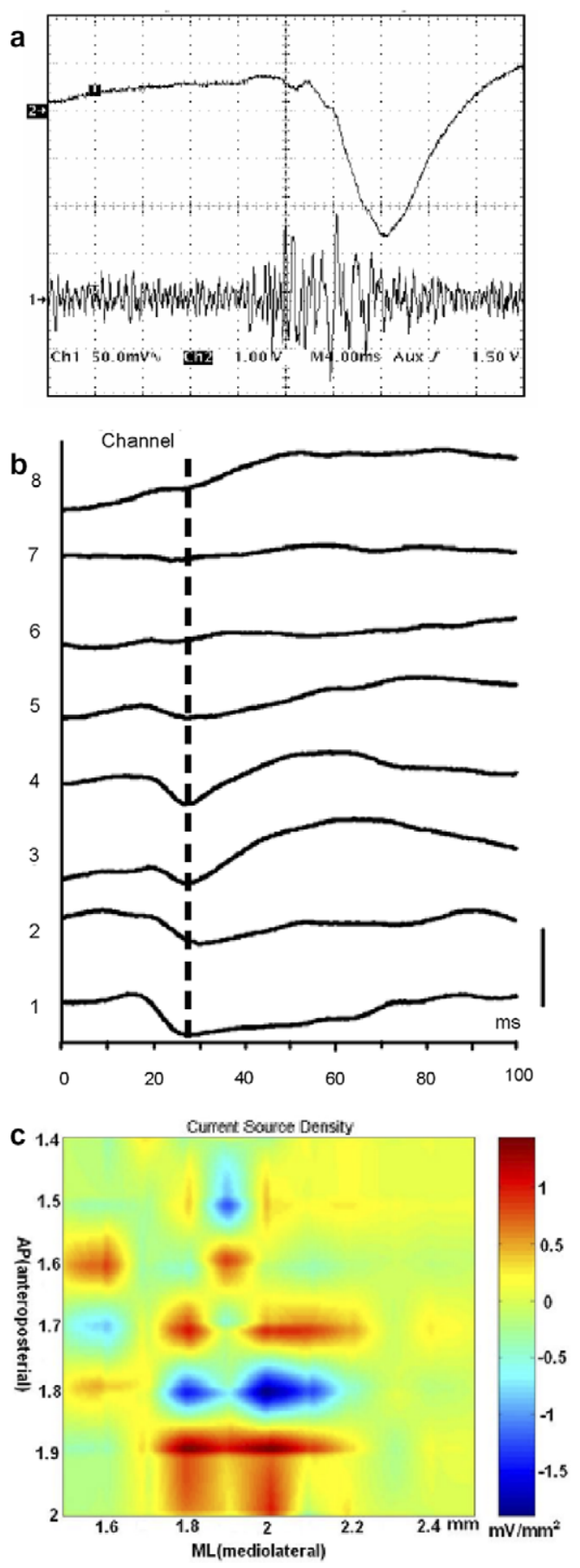

Fig. 5. An example of mapping. (a) The FP (upper trace) and MUA (lower trace) detected by the same electrode can be recorded at the same time. (b) Simultaneous recording of the eight-channel FPs in the cerebral cortex of a Wistar rat. The middle portion of the rat tail was stimulated by an electrical stimulator (Digitimer DS2) at $1 \mathrm{~Hz}$. The vertical calibration bar represents $500 \mu \mathrm{V}$ for all the waveforms. (c) A 2D CSD distribution of the response at $29 \mathrm{~ms}$ latency (the dashed line in Fig. 5b) was plotted. The responses were recorded at a depth of $500 \mu \mathrm{m}$ from the surface of the cortex.
Butterworth low-pass filter, with the actual passband from 0.18 to $110 \mathrm{~Hz}$; the latter is that of the Bessel band-pass filter, with the actual pass-band from $280 \mathrm{~Hz}$ to $3.8 \mathrm{kHz}$. These gains were slight deviations from our original design, but remained within the acceptable limits.

A recording trial is illustrated in Fig. 5. The traces (hardcopied from the screen of the oscilloscope) show the FP and MUA data recorded by the same electrode. The eight-channel cortical FPs (as shown in Fig. 5b) were recorded 2.0 and $1.8 \mathrm{~mm}$ lateral and posterior to the bregma, respectively. The recordings were carried out $500 \mu \mathrm{m}$ below the surface of the cortex. The current source density (CSD) distribution obtained by performing a Laplacian operation on the FPs is shown in Fig. 5c. The CSD mapping shows a current sink 1.8 and $\sim 1.8-2.1 \mathrm{~mm}$ posterior and lateral to the bregma, respectively, so the active center at this point could be approximated by a dipole with the source $0.1 \mathrm{~mm}$ posterior to the sink.

\section{Discussion}

Multichannel microelectrodes have been developed for various applications during the past three decades. Some previous studies have focused on using microwires as the electrode [23-25]. Recent advances in micro-electro-mechanical systems (MEMS) technologies have promoted the use of silicon-based probes for neuroscience research, as is done with the Michigan neural probes, and thus, increasing the demand of multichannel recording system.

There are many ways in which to implement a multichannel signal-conditioning unit. Usually, several stages are adopted for noise reduction. With high performance in mind, three stages were used in our design, each of which showed excellent flexibility. For example, the battery powered headstage could be placed within the Faraday's cage and the preamplifier could drive a cable longer than $10 \mathrm{~m}$ with no deterioration of the signals. For the electrophysiological characteristics, the maximum flatness Butterworth filter and constant group delay Bessel filter are implemented, respectively. Moreover, the new parallel architecture of the filter stage allowed the MUAs and FPs to be recorded simultaneously without any extra signal processing.

In addition to maximizing the performance-tocost ratio of the conditioning unit, pseudo-synchronous acquisition is a novel concept that highlights 
the problems of using a conversional multiplexing method to acquire multichannel neuronal signals that are highly correlated to each other. Burst multiplexing or pseudo-synchronous acquisition appears to limit the errors of channels. This idea is a compromise between hardware complexity and cost, and is an affordable approach at present. Here, this equipment, including the LabVIEW environment, was purchased, not only to save time but also to take advantage of its user friendly interface.

The progress of electrophysiology relies heavily on advances in new recording technologies. Multichannel simultaneous recording of the neuronal ensemble is a promising method. In order to function properly, this new trend requires complex and expensive systems, such as those currently produced by Plexon Inc. (Multichannel Acquisition Processor $^{\mathrm{TM}}$, Texas, USA) and Axon Instruments (California, USA). However, these systems are usually too expensive for laboratories with limited financial budgets. Furthermore, most they are specifically designed for use in MUA recording and analysis, and the accompanying programs cannot be modified for use in other applications, such as currentsource-density analysis. The proposed system is designed for FPs as well as MUA recordings. It is cost effective, reliable and provides the flexibility of a modular design. Both the hardware circuits and the analytical programs can be added as required. Although this might not be the optimal system, it is workable and cost effective. Thus, the proposed system should be useful for neuroscientists who want to set up their own system.

\section{Acknowledgements}

We thank Mr. Y.-C. Tsai, C.-M. Lin, T.-K. Teng, and Ms. M.-Y. Lu for their help in executing some parts of this project. Our study was supported by grants NSC93-2213-E-002-066.

\section{References}

[1] M.A.L. Nicolelis, L.A. Baccala, R.C.S. Lin, J.K. Chapin, Sensorimotor encoding by synchronous neural ensemble activity at multiple levels of the somatosensory system, Science 268 (2) (1995) 1353-1358.

[2] A.A. Ghazanfar, C.R. Stambaugh, M.A.L. Nicolelis, Encoding of tactile stimulus location by somatosensory thalamocortical ensembles, J. Neurosci. 20 (10) (2000) 37613775 .

[3] M.A.L. Nicolelis, A.A. Ghazanfar, B. Faggin, S. Votaw, L.M.O. Oliverira, Reconstructing the engram: simultaneous multiple site, many single neuron recordings, Neuron 18 (1997) 529-537.

[4] J.D. Kralik, D.F. Dimitrov, D.J. Krupa, D.B. Katz, D. Cohen, M.A.L. Nicolelis, Techniques for long-term multisite neuronal ensemble recordings in behaving animals, Methods 25 (2001) 121-150.

[5] T.P.S. Powell, V.B. Mountcastle, Some aspect of the function organization of the cortex of the postcentral gyrus of the monkey: a correlation of findings obtained in a single unit analysis with cytoarchitecture, Bull. John Hopkins Hosp. (1959) 105.

[6] J. Schouenborg, J. Kalliomaki, P. Gustavsson, I. Rosen, Field potential evoked in rat primary somatosensory cortex (SI) by impulses in cutaneous $\mathrm{A}_{\beta^{-}}$and $\mathrm{C}$-fibers, Brain Res. 397 (1986) 86-92.

[7] G. Buzsaki, Large-scale recording of neuronal ensembles, Nature Neurosci. 7 (5) (2004) 446-451.

[8] Y.-Y. Chen, T.-S. Kuo, F.-S. Jaw, A laser micromachined probe for recording multiple field potentials in the thalamus, J. Neurosci. Meth. 139 (2004) 99-109.

[9] F.-Z. Shaw, R.-F. Chen, H.-W. Tsao, C.-T. Yen, A multichannel system for recording and analysis of cortical field potentials in freely moving rats, J. Neurosci. Meth. 88 (1999) 33-43.

[10] K.S. Guillory, R.A. Normann, A 100-channel system for real time detection and storage of extracellular spike waveforms, J. Neurosci. Meth. 91 (1999) 21-29.

[11] I. Szabo, A. Czurko, J. Csicsvari, H. Hirase, X. Leinekugel, G. Buzsaki, The application of printed circuit board technology for fabrication of multi-channel micro-drives, J. Neurosci. Meth. 105 (2001) 105-110.

[12] A.C. Hoogerwerf, K.D. Wise, A three-dimensional microelectrode array for chronic neural recording, IEEE Trans. Biomed. Eng. 41 (12) (1994) 1136-1146.

[13] A.L. Owens, T.J. Denison, H. Versnel, M. Rebbert, M. Peckerar, S.A. Shamma, Multi-electrode array for measuring evoked potentials from surface of ferret primary auditory cortex, J. Neurosci. Meth. 58 (1995) 209-220.

[14] J.C. Williams, R.L. Rennaker, D.R. Kipke, Long-term neural recording characteristics of wire microelectrode arrays implanted in cerebral cortex, Brain Res. Protoc. 4 (3) (1999) 303-313.

[15] A. Bragin, J. Hetke, C.L. Wilson, D.J. Anderson Jr., J. Engel, G. Buzsaki, Multiple site silicon-based probes for chronic recordings in freely moving rats: implantation, recording and histological verification, J. Neurosci. Meth. 98 (2000) 77-82.

[16] C.J. Richard, Tutorial: analog data acquisition technology Part III- sample-and-holds, instrumentation amplifier, and analog multiplexers, IEEE Micro (1982) 20-35.

[17] J.L. Novak, B.C. Wheeler, A high-speed multichannel neural data acquisition system for IBM PC compatibles, J. Neurosci. Meth. 26 (1989) 239-247.

[18] F.-S. Jaw, C.-W. Lin, C.-Y. Wang, Sampling variation caused by A/D cards due to external trigger, J. Neurosci. Meth. 61 (1995) 175-178.

[19] C.-N. Chien, J.-K. Jang, F.-S. Jaw, Study on a 'pseudo' synchronous A/D card for the mapping of multi-channel signals, in: Conf. Biomed. Eng. Technol., Taiwan, 2000.

[20] M.R. Neuman, Biopotential amplifiers, in: J.G. Webster (Ed.), Medical instrumentation application and design, third ed., John Wiley \& Sons, New York, 1998, pp. 233-286. 
[21] H.W. Smit, K. Verton, C.A. Grimbergen, A low-cost multichannel preamplifier for physiological signals, IEEE Trans. Biomed. Eng. 34 (4) (1987) 307-310.

[22] F.-S. Jaw, Optimal sampling of electrophysiological signals, Neurosci. Res. Commun. 28 (2001) 75-84.

[23] C.A. Palmer, A microwire technique for recording single neurons in unrestrained animals, Brain Res. Bull. 5 (1978) $422-424$.
[24] J.L. Kubie, A driveable bundle of microwires for collecting single-unit data from freely moving rats, Physiol. Behav. 32 (1984) 115-118.

[25] D. Banks, B. Matthews, The use of microwires for chronic recording from the thalamus in cats, J. Physiol. 401 (1988) 20. 